Article

\title{
Reusable, Non-Invasive, and Ultrafast Radio Frequency Biosensor Based on Optimized Integrated Passive Device Fabrication Process for Quantitative Detection of Glucose Levels
}

\author{
Yang Li ${ }^{1,2,+}$, Zhao Yao ${ }^{3,+}\left(\mathbb{D}\right.$, Wenjing Yue ${ }^{1,2, *}$, Chunwei Zhang ${ }^{1,2}$, Song Gao ${ }^{1,2, *(\mathbb{D})}$ \\ and Cong Wang 4 \\ 1 School of Information Science and Engineering, University of Jinan, Jinan 250022, China; \\ ise_liy@ujn.edu.cn (Y.L.); ise_zhangcw@ujn.edu.cn (C.Z.) \\ 2 Shandong Provincial Key Laboratory of Network Based Intelligent Computing, University of Jinan, \\ Jinan 250022, China \\ 3 College of Microtechnology \& Nanotechnology, Qingdao University, Qingdao 266071, China; \\ yao9074@hotmail.com \\ 4 Department of Microwave Engineering, Harbin Institute of Technology, Harbin 150001, China; \\ kevinwang@hit.edu.cn \\ * Correspondence: ise_yuewj@ujn.edu.cn (W.Y.); ise_gaos@ujn.edu.cn (S.G.) \\ + These authors contributed equally to this work.
}

Received: 18 January 2020; Accepted: 9 March 2020; Published: 11 March 2020

\begin{abstract}
The increase in the number of people suffering diabetes has been the driving force behind the development of glucose sensors to overcome the current testing shortcomings. In this work, a reusable, non-invasive and ultrafast radio frequency biosensor based on optimized integrated passive device fabrication process for quantitative detection of glucose level was developed. With the aid of the novel biosensor design with hammer-shaped capacitors for carrying out detection, both the resonance frequency and magnitude of reflection coefficient can be applied to map the different glucose levels. Meanwhile, the corresponding fabrication process was developed, providing an approach for achieving quantitative detection and a structure without metal-insulator-metal type capacitor that realizes low cost and high reliability. To enhance the sensitivity of biosensor, a 3-min dry etching treatment based on chlorine/argon-based plasma was implemented for realizing hydrophilicity of capacitor surface to ensure that the biosensor can be touched rapidly with glucose. Based on above implementation, a non-invasive biosensor having an ultrafast response time of superior to $0.85 \mathrm{~s}$, ultralow LOD of $8.01 \mathrm{mg} / \mathrm{dL}$ and excellent reusability verified through five sets of measurements are realized. The proposed approaches are not limited the development of a stable and accurate platform for the detection of glucose levels but also presents a scheme toward the detection of glucose levels in human serum.
\end{abstract}

Keywords: radio frequency resonator; non-invasive glucose biosensor; integrated passive device fabrication process; quantitative detection; reusable biosensor; ultrafast biosensor

\section{Introduction}

Diabetes is a group of lifelong metabolic diseases caused by multiple etiologies, which is characterized by chronic hyperglycemia. Human in hyperglycemic for long-term will lead to microvascular damage and endanger the heart, brain, kidney, peripheral nerves, eyes, feet, etc. [1]. According to the World Health Organization statistics, diabetes, having more than 100 diabetes complications is one of the most complicated diseases. It is known that more than half of diabetic deaths 
are caused by cardiovascular and cerebrovascular diseases, and $10 \%$ are caused by nephropathy [2-5]. Patients with amputation due to diabetes are 10 to 20 times more numerous than non-diabetic patients. Clinical data show that 30 40\% of patients have at least one complication after the onset of diabetes around 10 years, and drug treatment is difficult to recover since complications occur [6]. Hence, it is crucial to develop a method to realize accurate glucose detection for treatment and management of diabetes. The glucose biosensing detection system composed of bio-sensing detection conversion and peripheral electronic systems can be applied to monitor the glucose concentration of diabetic patients in time. In particular, biosensing detection conversion plays a very important role in the sensing system, which determines several key factors, such as accuracy, detection time and system cost [7-9]. A biosensor can convert different concentrations of glucose into corresponding electrical signals for output, which has advantages of high accuracy, fast analysis, low cost, good repeatability, simple operation and high specificity by comparing with traditional detection methods [10-16]. With the aid of chips, microfluidics systems and labs-on-a-chip, biosensor technology have developed rapidly and can be divided into electrochemical biosensors and optical biosensors based on the type of signal conversion [17-26]. Electrochemical biosensors enable the detection of biomarkers by adding specific enzymes on the electrodes, which has many advantages, such as high sensitivity, simple operation and cost-effective [27-29]. However, the introduction of some small molecule exotic vectors acting as channels between enzymes and motors electrodes leads to a slowing reaction, a decreasing performance, a deteriorating reliability and frequent replacement. Moreover, another key factor limiting the application of electrochemical sensors is the fact that the electrolytes need to be replenished on a regular basis, which significantly increase burden to subsequent costs [30-32]. With regard to optical biosensors, it needs a long settling time for detection and is highly susceptible to change the test results for ambient light [33-41]. Recently, a biosensor based on radio frequency (RF) techniques has attracted enormous attention and is regarded as a promising and competitive candidate for implementing third-generation glucose biosensors [42-47]. Compared to other types of biosensors, RF biosensors offer the following advantages: first, they are different from the electrochemical biosensors, which are subject to the constraints of the use environment and performance degradation with service time. The RF biosensors are not susceptible to environmental factors such as external light, temperature, humidity, etc., and can maintain stability in long-term complex environments. Secondly, The RF biosensor can perform rapid detection of biomarkers in real time without the need for pre-stabilization time. Thirdly, the detection time of the RF biosensor only depends on the sweep period of the vector network analyzer, exhibiting an evident advantage in short detection time. Fourthly, there is no need to add exotic vectors to mark for sensing and a label-free detection is achieved by dropping the determinand to the RF biosensor detection area.

In this work, we developed a non-invasive RF glucose sensor based on quantitative detection. Benefiting from the novel biosensor design with hammer-shaped capacitors, it was found that the concentrations of glucose are proportional to the biosensor resonance frequency and magnitude of reflection coefficient, which implies that both the resonance frequency and the magnitude of reflection coefficient can be applied to map the different glucose levels, indicating the feasibility of the proposed biosensor in glucose detection. Moreover, the corresponding fabrication process has been further elaborated, holding several merits such as that it can provide a volume-fixed passivation layer for quantitative detection and non-MIM (metal-insulator-metal)-type capacitor for reducing fabrication procedure with low cost and high reliability. To enhance the sensitivity of a biosensor, a dry etching treatment was implemented by using chlorine/argon-based plasma for realizing hydrophilicity of capacitor surface to ensure that the biosensor can be touched rapidly with glucose. Ultimately, a non-invasive biosensor with a memorable response time, ultralow limit of detection (LOD) and excellent reusability were realized and verified through measurements. 


\section{Materials and Methods}

\subsection{Biosensor Operating Mechanism}

The scanning electron microscope (SEM) image of the proposed glucose biosensor based on RF resonator is illustrated in Figure 1a, which is composed of two spiral inductors and two hammer-shaped capacitors. An embedded structure was skillfully introduced to minimize the dimensions of the overall circuit. To form inductor-capacitor (L-C) resonance tanks, two capacitors were placed in parallel on two inductors and two common ports for connecting inductor and capacitor were defined as ground. For the detailed parameters of the proposed pattern, the two inductors metal lines were $15 \mu \mathrm{m}$ in width, the turns were 4.5, the inner diameter was $610 \mu \mathrm{m}$, and the spacing of the metal line is 15 $\mu \mathrm{m}$. As regards the hammer-shaped capacitors, it consisted of two rectangles with a length of 300 $\mu \mathrm{m}$ and a width of $95 \mu \mathrm{m}$. In order to form a capacitor, the distance between the two rectangles was set to $20 \mu \mathrm{m}$. After dicing, the total area of the chip was $1060 \mu \mathrm{m} \times 2380 \mu \mathrm{m}$. The microwave propagation theory can be applied to analyze the biosensor operating mechanism, described as follows. The RF signal is initiated at the input port, transmitted along the middle straight transmission line, partially attenuated at both L-C-type resonators, ending at the output port. Specifically, the two hammer-shaped capacitors are the key parts of detection, which are employed to alter the capacitive effect. As shown in Figure 1b, when the glucose samples are added onto the circuit, the performance of the circuit will be changed due to the alterative capacitance, that is, because different glucose concentrations have a different permittivity to affect the capacitance. Therefore, glucose concentrations at different levels can be detected by analyzing relevant variation of RF parameters, such as magnitude of reflection coefficient and frequency shift, indicating that such a chip can be used clinically to diagnose blood glucose concentrations in diabetic patients in real time. Then, an electric field distribution of the proposed biosensor is simulated based on an Advanced Design System Momentum Microwave simulator at the resonance frequency, as shown in Figure 1c. It can be observed that the maxima of the electric field is located along two inner capacitors, which means that the electric coupling of the two capacitors plays the dominant role in the resonance frequency, validating the correctness of the above analysis. Figure $1 \mathrm{~d}$ shows the equivalent circuit modelling of the circuit. It can be observed that the equivalent circuit consists of two L-C resonance tanks, which, respectively, correspond to the resonance tank composed of a spiral inductor and a hammer-shaped capacitor in parallel. With regard to the detailed symbols, they are depicted as follows: $L_{T}, R_{T}, C_{L}, R_{C L}, L_{L}, R_{L L}, C_{R}, R_{C R}, L_{R}$, $R_{L R}$ and $C_{S U B}$ represent the inductance of the middle transmission line of the biosensor, the resistive loss of the middle transmission line of the biosensor, the capacitance of the left-side hammer-shaped capacitor, the resistive loss of the left-side hammer-shaped capacitor, the inductance of the left-side spiral inductor, the resistive loss of the left-side spiral inductor, the capacitance of the right-side hammer-shaped capacitor, the resistive loss of the right-side hammer-shaped capacitor, the inductance of the right-side spiral inductor, the resistive loss of the right-side spiral inductor, and the capacitances associated with the substrate, respectively. The resonance frequency of the proposed biosensor can be described by [48]

$$
f=\frac{1}{2 \pi \sqrt{L C}}
$$

where $C$ is equal to $C_{L}$ and $C_{R}$, which is determined by

$$
C=\frac{1}{2} w\left(\frac{\varepsilon_{d} \varepsilon_{0} \varepsilon_{r}}{9.6}\right)^{0.8}\left(\frac{s}{w}\right)^{m_{0}} \exp \left(k_{0}\right)-3 w\left(\frac{\varepsilon_{d} \varepsilon_{0} \varepsilon_{r}}{9.6}\right)^{0.9}\left(\frac{s}{w}\right)^{m_{e}} \exp \left(k_{e}\right)
$$

With

$$
\begin{gathered}
m_{0}=\frac{w}{h}[0.619 \log (w / h)-0.3853] \\
k_{0}=4.26-1.453 \log (w / h) \\
m_{e}=0.8675
\end{gathered}
$$




$$
k_{e}=2.043\left(\frac{w}{h}\right)^{0.12}
$$

where $w$ is the hammer-shaped capacitor width, $h$ is the thickness of GaAs substrate, $s$ is the space between two capacitors, $\varepsilon_{0}$ is the free-space permittivity, $\varepsilon_{r}$ is permittivity of GaAs substrate, and $\varepsilon_{d}$ is the permittivity of the determinant. Based on Equations (2)-(6), the resonance frequency of proposed biosensor can be reproduced as

$$
f=\frac{1}{2 \pi} L^{-\frac{1}{2}}\left(1 / 2 w\left(\frac{\varepsilon_{d} \varepsilon_{0} \varepsilon_{r}}{9.6}\right)^{0.8}\left(\frac{s}{w}\right)^{m_{0}} \exp \left(k_{0}\right)-3 w\left(\frac{\varepsilon_{d} \varepsilon_{0} \varepsilon_{r}}{9.6}\right)^{0.9}\left(\frac{s}{w}\right)^{m_{e}} \exp \left(k_{e}\right)\right)^{-\frac{1}{2}}
$$

Since the design topology is fixed, the value of $L, w, \mathrm{~s}, m_{0}, k_{0}, m_{e}, k_{e}, \varepsilon_{r}$ is determined accordingly. Therefore, it can be inferred that the resonance frequency of the proposed biosensor is proportional to the permittivity of glucose solution $\varepsilon_{d}$. With regard to the reflection coefficient, it is mainly depended on the match between input impedance and characteristic impedance. In this work, the input impedance is fixed at $50 \Omega$, and a low reflection coefficient will be obtained if characteristic impedance $Z_{c}$ gets closer to $50 \Omega$. Here,

$$
\begin{gathered}
Z_{c}=\frac{\eta}{2 \pi \sqrt{\varepsilon_{r}}} \operatorname{In}\left(\frac{8 h}{w}+0.25 \frac{w}{h}\right) \\
\eta=\frac{12 \pi}{5} \varepsilon_{d} \cdot Z_{0}
\end{gathered}
$$

where $\eta$ is the wave impedance in the glucose solution and $Z_{0}$ is the source/load impedance, the value of which is $50 \Omega$. Based on Equations (8) and (9), the characteristic impedance $Z_{c}$ can be reproduced as

$$
Z_{c}=\frac{6 \varepsilon_{d} \cdot Z_{0}}{5 \sqrt{\varepsilon_{r}}} \operatorname{In}\left(\frac{8 h}{w}+0.25 \frac{w}{h}\right)
$$

Based on Equation (10), we can conclude that the different glucose concentrations can also be reflected by the variation of reflection coefficient.

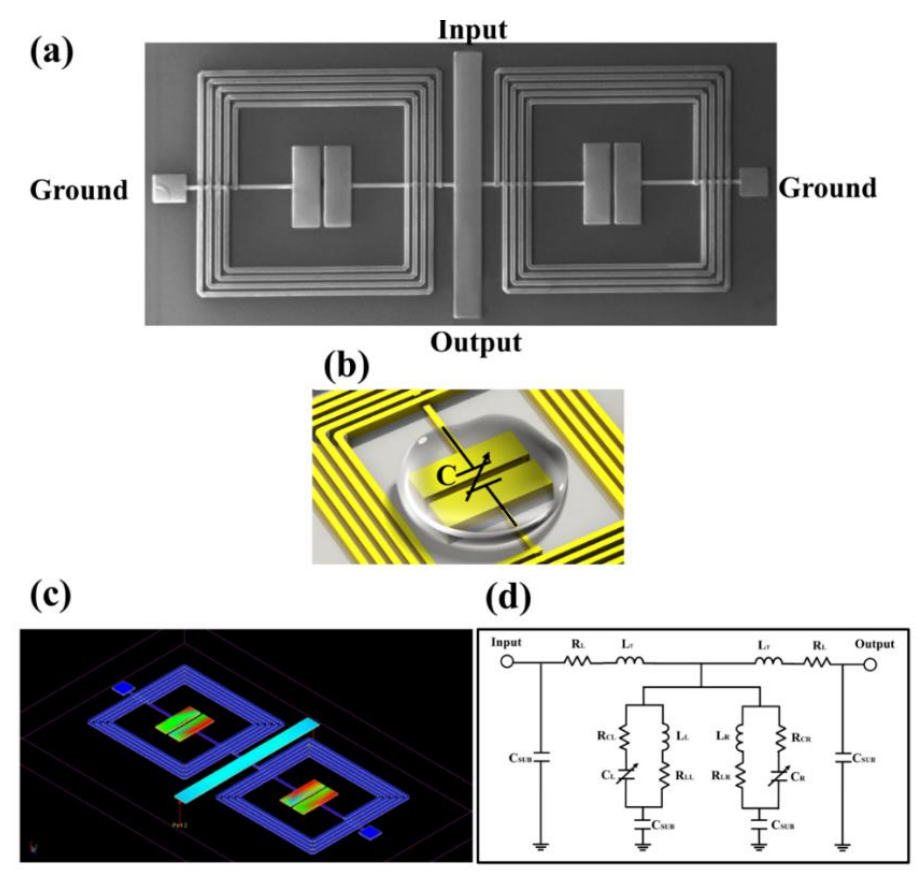

Figure 1. The illustration of glucose biosensor based on the RF resonator. (a) The SEM image of the fabricated biosensor with ports description. (b) The sensitive area of the hammer-shaped capacitor. (c) The simulated electric field distribution for the proposed biosensor at the resonance frequency.

(d) The equivalent circuit model of the proposed biosensor. 


\subsection{Biosensor Fabrication Techniques}

This section provides a detailed explanation to the proposed biosensor fabrication processing, which is an optimized process of the traditional integrated passive device process [49,50], exploiting the hammer-shaped capacitors instead of metal-insulator-metal (MIM) capacitors. The proposed implementation not only avoids the multi-processes realization of silicon nitride $\left(\mathrm{SiN}_{\mathrm{x}}\right)$ based on plasma enhanced chemical vapor deposition (PECVD) method for a cost-effective fabrication, it also effectively averts the introduction of the low breakdown voltage of MIM capacitors, which is an upgrade on the reliability of the biosensor. In this work, a GaAs wafer with a relative permittivity of 12.85 and loss tangent of 0.002 was selected to fabricate biosensor, which can avoid parasitic capacitance and inductive loading of conductive substrates. The proposed fabrication process starts from substrate cleaning with an ultrasonic acetone bath ( $5 \mathrm{~min}$ ), implementing isopropyl alcohol (IPA) treatment (1 min), and DI water flushing (5 min), respectively (Step 1). A passivation layer was then deposited by PECVD, comprising $\mathrm{SiN}_{\mathrm{x}}$ with a relative dielectric constant of 7.5 and a loss tangent of 0.002 (step 2). The deposition was carried out at a ratio of $\mathrm{SiH}_{4}$ and $\mathrm{NH}_{3}$ of $320: 9 \mathrm{sccm}$ at a chamber pressure of $1200 \mathrm{mTorr}$, a chamber temperature of $250{ }^{\circ} \mathrm{C}$, a $100 \mathrm{~W}$ RF power and a $2000 \mathrm{sccm}$ gas flow. The entire process time was fixed to $400 \mathrm{~s}$ to get a target thickness of $0.2 \mu \mathrm{m}$. The deposited $\mathrm{SiN}_{\mathrm{x}}$ layer is necessary to obtain a uniform surface on the defects and roughness of the substrate. The deposited $\mathrm{SiN}_{\mathrm{x}}$ passivation layer also promotes adhesion between the bottom metal layer and the GaAs substrate. A photolithography process of the bottom metal, which involves photoresist spin coating with edge bead removal, exposure with the aid of mask, development, inspection, and critical dimension (CD) measure, was performed after $\mathrm{SiN}_{\mathrm{x}}$ deposition (Step 3). According to the specific bottom metal shape desired, this work chose to perform photolithography with a negative photoresist (NR9-3000PY, Futurrex, Franklin, NJ, USA). Then a 2- $\mu \mathrm{m}$-thick Au bottom metal layer was formed using by the sputtering deposition method (Step 4). The deposition process was performed at a pressure of $7.5 \mathrm{mTorr}$ and with a minimum deposition rate of $0.5 \mathrm{~A} / \mathrm{s}$. In addition, the room temperature sputtering method was performed to achieve a better metal roughness with a RMS value of $1.93 \mathrm{~nm}$, the purpose of which is to reduce the possibility of a short circuit between the bottom metal and the top metal. Then a photoresist liff-off process was carried out by spraying acetone (Step 5).

Next, the forming process on the air-bridge post of spiral inductor was implemented (Step 6). First, the photolithography process, including photoresist spin coating, exposure with the aid of mask, development, inspection, $C D$ measure, was performed using the negative photoresist, which is the same as Step 3. Then, for the purpose of constructing an arch-bridge structure, a hard-baking process followed by a photolithography process was performed, whose baking time and baking temperature were chosen as $180 \mathrm{~s}$ and $130^{\circ} \mathrm{C}$, respectively. Next, a Ti seed metal layer was grown by the sputtering deposition method to a thickness of $1000 \mathrm{~nm}$ (Step 7). An additional $30 \mathrm{~s}$ sputter-etching process was performed prior to Ti seed metal deposition so as to reduce the probability of occurrence of peeling off between top layer and bottom layer. Then, a top metal layer photolithography process (Step 8) and a top metal deposition (Step 9) were carried out, which are the same as step 3 and step 4. A $2 \mu \mathrm{m}$ top metal layer was deposited using the sputtering method based on Au material, which can prevent moisture and oxidation of glucose due to its chemical stability. Subsequently, a photoresist liff-off process was carried out by spraying acetone, as in process step 5 (Step 10). Finally, a $2 \mu \mathrm{m} \mathrm{SU-8} \mathrm{final} \mathrm{passivation}$ layer was patterned for quantitative detection to reduce the interference of the glucose solution toward the inductor and transmission-line area (Step 11). Figure 2 illustrates the whole fabrication process flow as explained above, and the inset shows SEM images of the fabricated inductor and capacitor. It should be noted that $\mathrm{Au} \mathrm{w}$ chosen as the structural layer in this fabrication process, the purpose of which is as follows: first, it can prevent the metal layer from chemically reacting with substances such as glucose and water which introduce interference in repeat measurement. In addition, it is different from the conventional copper used as the metal layer; the utilization of Au layer can reduce unnecessary signal losses, which improves the transmission parameter by minimizing the signal loss. 


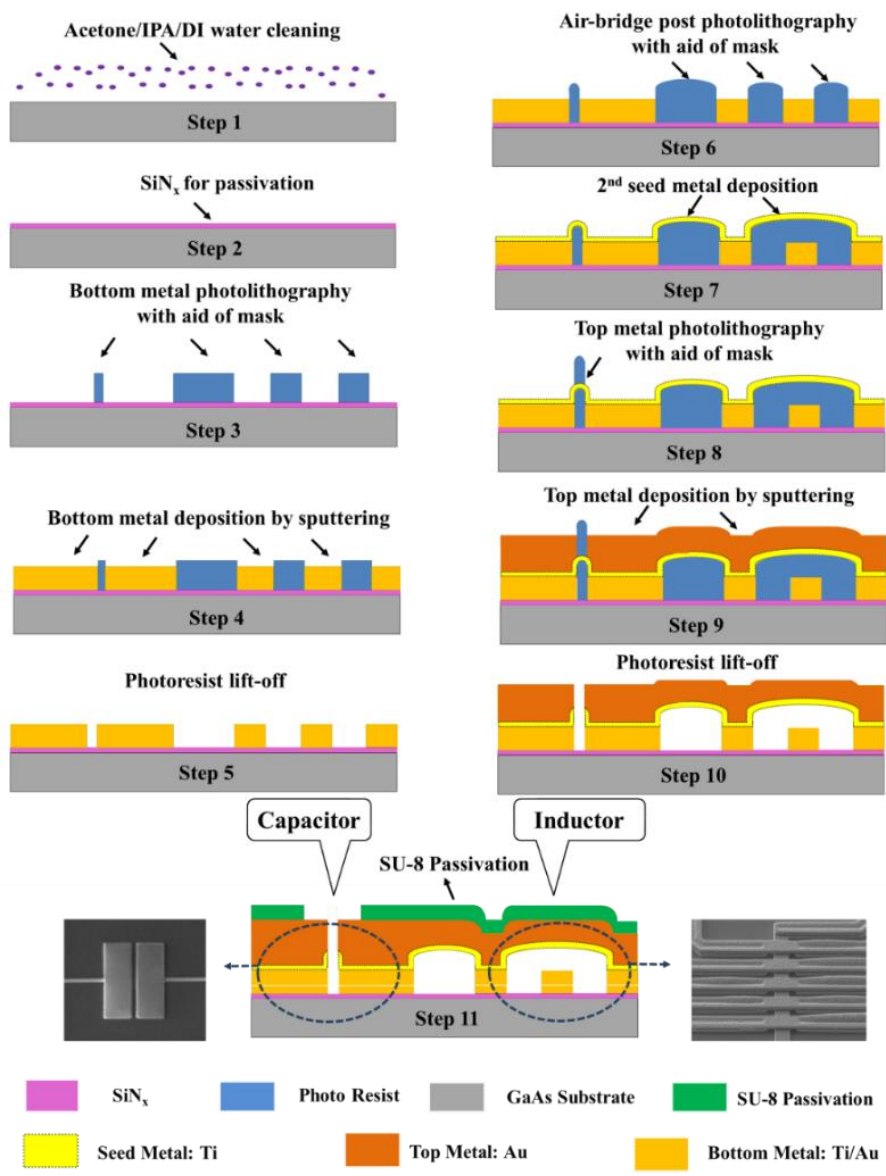

Figure 2. The fabrication process flow of the proposed biosensor.

\subsection{RF Detection Methods}

In order to perform the RF response measurements on the proposed biosensor, the measurement platform was successfully constructed and consisted of a vector network analyzer (VNA), a biosensor under test, a quantitative micro pipette, and glucose samples, as shown in Figure 3a. In this work, ten different glucose samples with a concentration range of 50 to $500 \mathrm{mg} / \mathrm{dL}$ were prepared, which were composed of a mixture of glucose anhydrose (Shanghai Macklin Biochemical Co., Ltd., Shanghai, China) and deionized water, and all analyzed $S$-parameters were measured using a Keysight FieldFox N9917A VNA. The proposed biosensor was attached on a printed circuit board (PCB) with two $50 \Omega$ transmission-line ports connecting from the input to output side and two bonding-wire connecting to ground signal, as depicted in Figure 3c. The relevant RF parameters of the bare chip were collected prior to testing the target substance; afterwards, the glucose solution of different concentrations was dropped onto the test area of the biosensor through a pipette with a fixed capacity of $0.5 \mu \mathrm{L}$, as illustrated in Figure $3 b$. Finally, the frequency responses were measured and recorded over a frequency range of 2-8 GHz. In order to eliminate the effects of temperature and humidity on the experimental results during the test, all the samples were measured at temperatures and relative humidity ranging from $22.5^{\circ} \mathrm{C}$ to $23.0^{\circ} \mathrm{C}$ and $45.5-45.8 \%$, respectively. After each RF measurement of the sample, the chip was first flushed several times with phosphate buffer saline (PBS, $\mathrm{pH}=6.9$, consisting of $137 \mathrm{mmol} / \mathrm{L} \mathrm{NaCl}$, $2.7 \mathrm{mmol} / \mathrm{L} \mathrm{KCl}, 10 \mathrm{mmol} / \mathrm{L} \mathrm{Na}_{2} \mathrm{HPO}_{4}$, and $2 \mathrm{mmol} / \mathrm{L} \mathrm{KH}_{2} \mathrm{PO}_{4}$ ) and then flushed with DI water prior to drying by air-blowing to ensure the glucose samples are completely removed such that the chip could be used repeatedly for multiple measurements. 


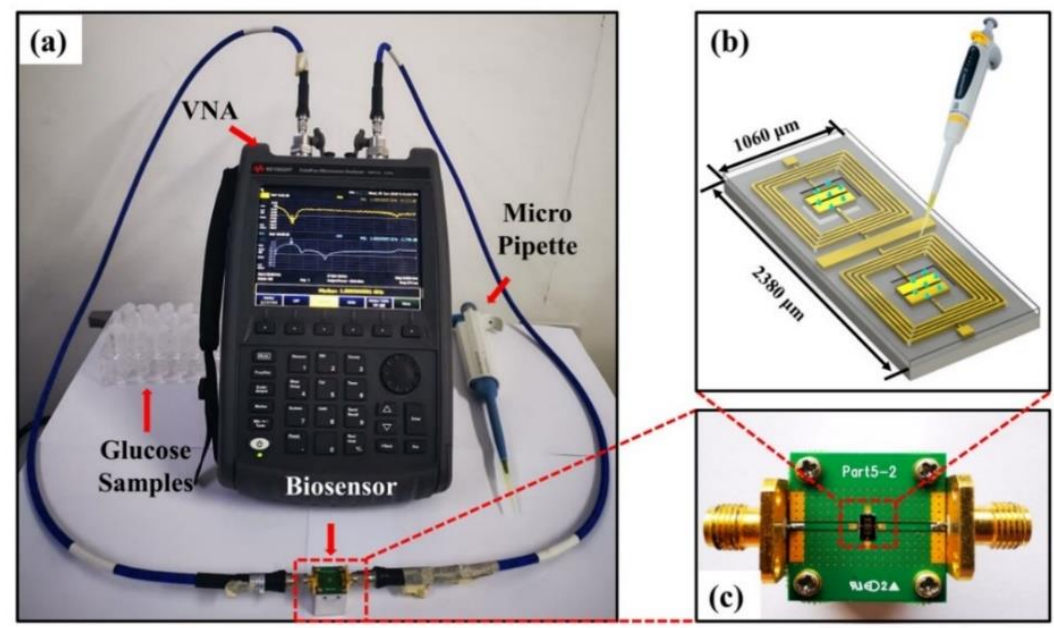

Figure 3. (a) The experimental setup of the proposed chip measurement for detecting variable glucose level. (b) The schematic diagram of measurement. (c) The fabricated biosensor attached on a PCB with input and output connection for glucose level measurement.

\section{Results and Discussions}

\subsection{Optimization of Sensitivity}

To enhance the sensitivity of the biosensor, a series of controlled dry etching processes based on $\mathrm{Au}$ layers were systematically carried out on the biosensor to investigate the effect between the roughness of surface and hydrophily hydrophobicity. Figure $4 \mathrm{a}-\mathrm{c}$ presents the surface roughnesses of the top $\mathrm{Au}$ layers measured by atomic force microscope (AFM) which are as-fabricated and etched based on $\mathrm{Cl}_{2} / \mathrm{Ar}$ plasmas processing with $3 \mathrm{~min}$ and $5 \mathrm{~min}$, respectively. The parameters of the Au dry etch process are shown in Table 1. As shown in Figure 4a, a smooth surface can be observed on the as-fabricated top layer with a root mean square (RMS) roughness of $2.03 \mathrm{~nm}$. When the chip is treated with $\mathrm{Cl}_{2} / \mathrm{Ar}$-based plasmas for $3 \mathrm{~min}$, the quality of surface roughness of top layer is deteriorated to $12.3 \mathrm{~nm}$, as depicted in Figure $4 \mathrm{~b}$. After dry etching for $5 \mathrm{~min}$, there is no obvious change on the surface roughness showing a RMS of $13.2 \mathrm{~nm}$. We can draw that the extra etching treatment, refering to over 3 min dry etching process, will not induce the Au roughness the deterioration any more after preliminary modification of the surface. Figure 4a.1-c.1 demonstrates the sessile drop data of glucose with $150 \mathrm{mg} / \mathrm{dL}$ on biosensor chip surface corresponding to above-mentioned three conditions. It can be observed that as-fabricated chip is highly hydrophobic with a glucose contact angle of $\sim 102.2^{\circ}$. While the contact angle decreases to $\sim 71.7^{\circ}$ and $\sim 70.1^{\circ}$ after dry etching for $3 \mathrm{~min}$ and $5 \mathrm{~min}$, respectively. The results show that the hydrophilicity of the biosensor increases as the surface roughness decreases, suggesting that the surface from hydrophobicity to hydrophilicity can be changed by the deteriorating surface roughness with plasma dry etching in order to promote the rate of fixation of glucose molecules and the resonance process.

Table 1. The recipe of Au dry etch process parameters.

\begin{tabular}{cc}
\hline \multicolumn{2}{c}{ Recipe Set Values } \\
\hline $\mathrm{Cl}_{2}$ & $20 \mathrm{sccm}$ \\
$\mathrm{Ar}$ & $5 \mathrm{sccm}$ \\
Pressure & $2 \mathrm{mTorr}$ \\
$\mathrm{RF}$ coil power & $600 \mathrm{~W}$ \\
$\mathrm{RF}$ platen power & $250 \mathrm{~W}$ \\
Temperature & $20^{\circ} \mathrm{C}$ \\
\hline
\end{tabular}




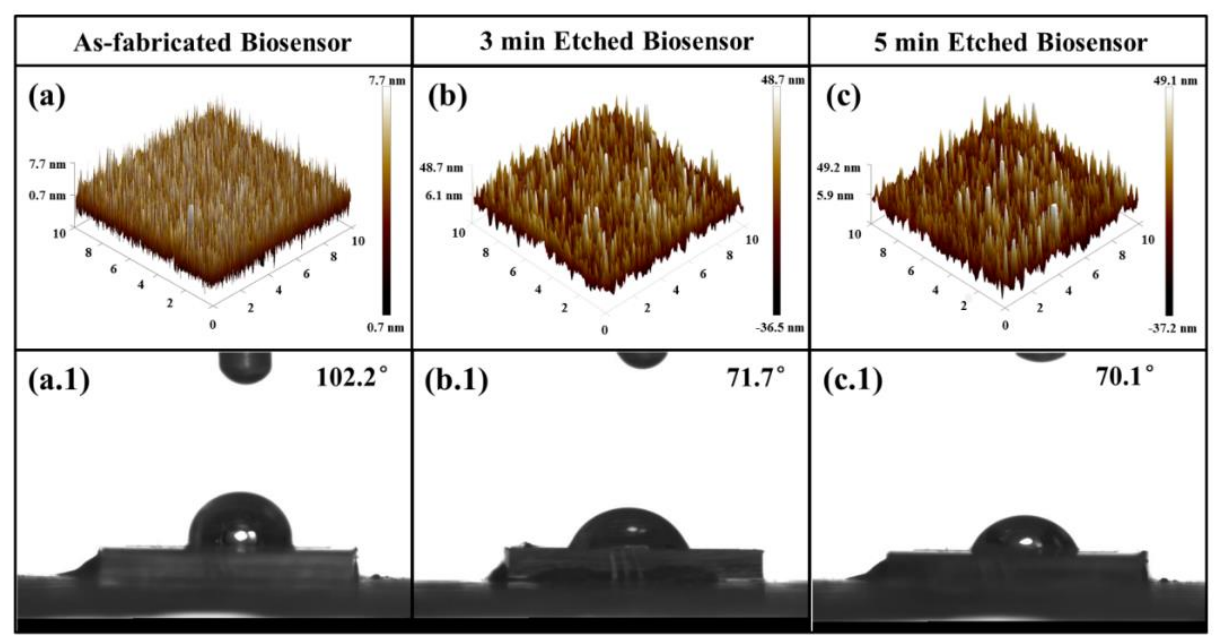

Figure 4. The AFM 3-D view of surface morphologies of top Au layers with (a) as-fabricated, (b) 3 min etched, and (c) $5 \mathrm{~min}$ etched. The images of $150 \mathrm{mg} / \mathrm{dL}$ glucose droplet onto biosensor with (a.1) as-fabricated, (b.1) 3 min etched, and (c.1) 5 min etched.

\subsection{Responses of Biosensor}

The responses of the proposed chip can be illustrated using RF parameters. In this work, we first measure the reflection coefficient and the resonance frequency under condition of the bare chip, the chip dropped using PBS solution, and the chip dropped using DI water, respectively. As shown in Figure 5a, the resonance frequency of bare chip is located at $6.30 \mathrm{GHz}$, and the other resonance frequencies revealed a downward shift with the instillation of PBS solution and DI water, which are located at $3.94 \mathrm{GHz}$ and $3.30 \mathrm{GHz}$, respectively. Meanwhile, magnitudes of the reflection coefficient show an increasing variation ranging from $-29.47 \mathrm{~dB}$ to $-12.18 \mathrm{~dB}$. The reasons for this can be explained as follows: the viscosity of DI water is lower than that of glucose solution and PBS, but conversely, the effective permittivity of DI water is greater than air, glucose solution, and PBS, resulting in the maximum frequency shift after dripping water onto the chip [51]. Then, a response experiment with different concentrations of glucose ranging from $50 \mathrm{mg} / \mathrm{dL}$ up to $500 \mathrm{mg} / \mathrm{dL}$ is explored. When the glucose solution is dropped on the surface of bare chip with increasing concentration, the resonance peaks regularly shift upward with an increasing of the magnitude of the reflection coefficient, as a result of the regular variation of effective permittivity, which is depicted in Figure 5b. It can be observed that the chip filled with glucose solution shows a tolerable resonance frequency shift in the range of 3.84-5.62 GHz accompanied with magnitude of reflection coefficient variation from $-14.18 \mathrm{~dB}$ to $-19.40 \mathrm{~dB}$. In comparison to the bare chip, the maximum resonance frequency shift occurs at a glucose level of $50 \mathrm{mg} / \mathrm{dL}$ with a shift of $2.46 \mathrm{GHz}$, and the maximum reflection coefficient variation comes up to $15.29 \mathrm{~dB}$ based on the same glucose level.

The relationships between glucose concentration and resonance frequency as well as glucose concentration and magnitude of reflection coefficient are depicted in Figure $5 c, d$, respectively. The regression analysis can be calculated as follows:

$$
\begin{aligned}
& Y_{1}[\mathrm{GHz}]=0.00413 \times X[\mathrm{mg} / \mathrm{dL}]+3.5801\left(\mathrm{R}^{2}=0.99216\right) \\
& \mathrm{Y}_{2}[\mathrm{~dB}]=0.01094 \times X[\mathrm{mg} / \mathrm{dL}]+13.32337\left(\mathrm{R}^{2}=0.9612\right)
\end{aligned}
$$

where $Y_{1}, Y_{2}$, and $X$ represent the resonance frequency, magnitude of reflection coefficient, and glucose concentration, respectively. Interestingly, a good linear correlation between glucose concentration and resonance frequency as well as glucose concentration and magnitude of reflection coefficient can be fitted, which unveils that both resonance frequency shift approach and magnitude of reflection 
coefficient variation approach can be applied to identify glucose level detection, paving a widening avenue for multi-parameter-sensitive detection of glucose.

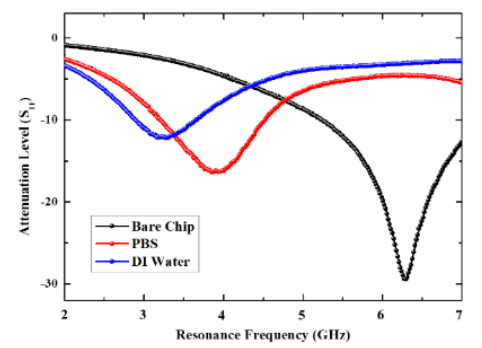

(a)

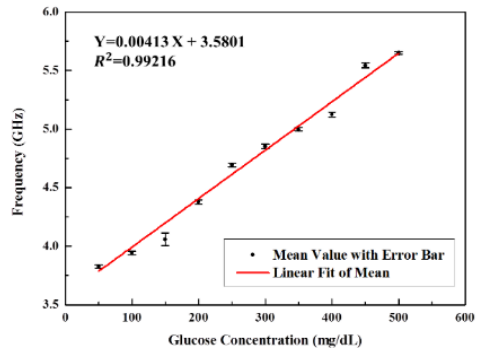

(c)

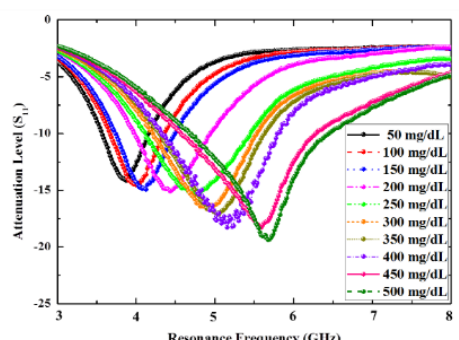

(b)

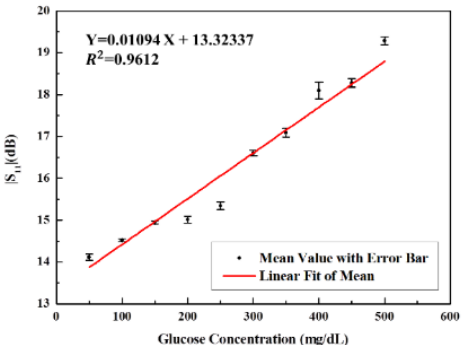

(d)

Figure 5. Shift in resonance frequency and magnitude of reflection coefficient $\left(\mathrm{S}_{11}\right)$ under different conditions: (a) various solution and (b) various glucose samples concentrations (50-500 mg/dL). Regression analysis for the shift in resonance frequency $(\mathbf{c})$ and magnitude of reflection coefficient $\left(\mathrm{S}_{11}\right)$ (d) with error bars for different glucose sample concentrations.

\subsection{Reusability, Real-Time Response Capability and LOD}

For the purpose of investigating the reusability of the proposed biosensor, the surface morphology of the sensing area of the chip, involving bare chip before test, chip added with $150 \mathrm{mg} / \mathrm{dL}$ glucose, and chip after cleaning, were characterized. Figure 6a,a.1,a.2, present the 2D and 3D view of the bare chip with an RMS value of $12.3 \mathrm{~nm}$ and a resonance frequency of $6.30 \mathrm{GHz}$, respectively. When the glucose solution was added to the chip with a concentration of $150 \mathrm{mg} / \mathrm{dL}$, an RMS value of $2.52 \mathrm{~nm}$ and a shifted resonance frequency of $4.07 \mathrm{GHz}$ can be achieved, which are shown in Figure 6b,b.1,b.2, respectively. The acquisition of such roughness compared with the bare chip is ascribed to the glucose particles coverage on uneven peaks and valleys of the surface. Interestingly, some cavities and wrinkles appear on the surface of the biosensor as a result of the uneven evaporation rate of the glucose solution. Then, the biosensor is rinsed with PBS and DI water. The RMS value of the biosensor after-cleaning was $11.7 \mathrm{~nm}$ (Figure 6c,c.1) and the resonance frequency returned to $6.30 \mathrm{GHz}$ (Figure 6c.2). From another perspective, the surface roughness profiles, before the test (Figure 6a.3) and after cleaning (Figure 6c.3), were found to be similar, which is obviously different from the conditions under the test (Figure 6b.3). The above-discussed results provide a good illustration that there is no chemical immobilization involved for the chip to create a resonance frequency and return the initial value after the test. Therefore, the proposed biosensor has a property of reusability for the future application.

The real-time response capability of the biosensor is very valuable for clinical medical applications. Therefore, based on the experimental setup shown in Figure 3a, we characterized the real-time response capability of a 3 min-etched chip by measuring the time at which the output waveform changes since glucose solution with concentration of $150 \mathrm{mg} / \mathrm{dL}$ is dropped onto the chip. It can be observed that the output waveform changes within $0.85 \mathrm{~s}$. The obtained quick response time demonstrates that the proposed biosensor has an excellent real-time response capability. Because of the limitation of 
the VNA scanning speed, which is $0.85 \mathrm{~s} /$ point, the real-time response of the biosensor is superior to $0.85 \mathrm{~s}$. With regard to the LOD, it is calculated from the following equation [52]:

$$
\mathrm{LOD}=3.3 \times \mathrm{SD} / \mathrm{m}
$$

where SD stands for the standard deviation of the frequency response and $m$ denotes the slope of the regression line. To obtain the standard deviation of the frequency response and slope of the regression line, the variations of resonance frequency during the five tests were measured, and the results are summarized in Table 2. With the aid of measurement, the LOD of $8.01 \mathrm{mg} / \mathrm{dL}$ was calculated. All relative standard deviations of less than $1 \%$ indicate a small discrete with respect to each resonance frequency. A comparative analysis based on RF techonology is summarized in Table 3, where the response time and LOD are superior to those achieved in previous studies.
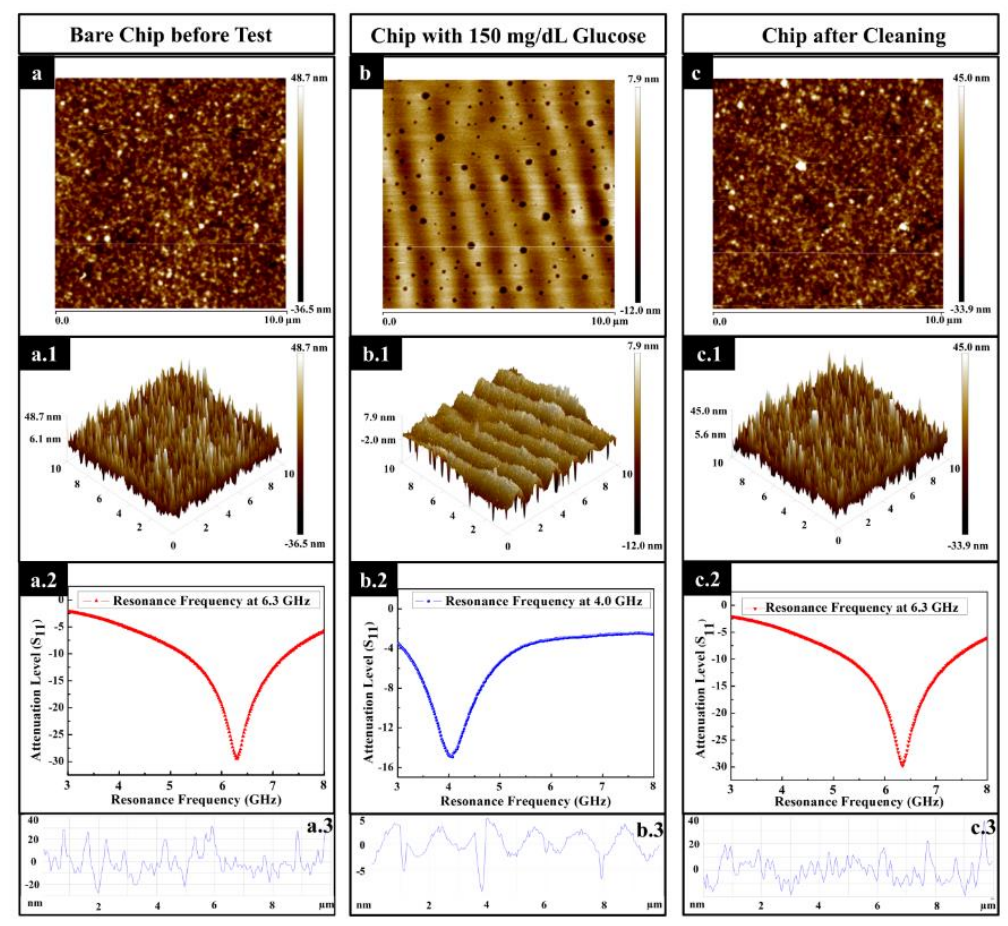

Figure 6. The analysis for surface morphology of the sensing area. (a) The $2 \mathrm{D}$ view of bare biosensor, (a.1) the 3D view of bare biosensor, (a.2) the RF measurement of bare biosensor, and (a.3) the cross-sectional surface line profile of surface morphology for bare biosensor. Bare chip refers to the chip treated by a 3 min dry etching process. (b) The 2D view of biosensor with glucose concentration of $150 \mathrm{mg} / \mathrm{dL}$, (b.1) the 3D view of biosensor with glucose concentration of $150 \mathrm{mg} / \mathrm{dL},(\mathbf{b} .2)$ the RF measurement of biosensor with glucose concentration of $150 \mathrm{mg} / \mathrm{dL}$, and (b.3) the cross-sectional surface line profile of surface morphology for biosensor with glucose concentration of $150 \mathrm{mg} / \mathrm{dL}$. (c) The 2D view of biosensor after cleaning, (c.1) the 3D view of biosensor after cleaning, (c.2) the RF measurement of biosensor after cleaning, and (c.3) the cross-sectional surface line profile of surface morphology for biosensor after cleaning. 
Table 2. Performances of the different measured resonance frequencies with various glucose concentrations ranging from $50 \mathrm{mg} / \mathrm{dL}$ to $500 \mathrm{mg} / \mathrm{dL}$.

\begin{tabular}{|c|c|c|c|c|c|c|c|c|c|}
\hline \multirow[b]{2}{*}{ S.N ${ }^{1}$} & \multirow{2}{*}{$\begin{array}{l}\text { G. C. }{ }^{2} \\
(\mathrm{mg} / \mathrm{dL})\end{array}$} & \multirow[b]{2}{*}{ 1st Test } & \multirow[b]{2}{*}{ 2nd Test } & \multicolumn{5}{|c|}{ Resonance Frequency (GHz) } & \multirow[b]{2}{*}{$\operatorname{RSD}^{5}(\%)$} \\
\hline & & & & 3rd Test & 4th Test & 5th Test & Mean $^{3}$ & $\begin{array}{c}\text { Mean } \pm \text { RSD } \\
\left(f_{a v}{ }^{4}\right)\end{array}$ & \\
\hline 1 & 50 & 3.83 & 3.84 & 3.81 & 3.83 & 3.82 & 3.826 & $3.826 \pm 0.26 \%$ & 0.26 \\
\hline 2 & 100 & 3.94 & 3.98 & 3.93 & 3.93 & 3.93 & 3.942 & $3.942 \pm 0.49 \%$ & 0.49 \\
\hline 3 & 150 & 4.08 & 4.03 & 4.06 & 4.05 & 4.07 & 4.058 & $4.058 \pm 0.42 \%$ & 0.42 \\
\hline 4 & 200 & 4.38 & 4.40 & 4.36 & 4.38 & 4.37 & 4.378 & $4.378 \pm 0.30 \%$ & 0.30 \\
\hline 5 & 250 & 4.72 & 4.68 & 4.67 & 4.70 & 4.70 & 4.694 & $4.694 \pm 0.37 \%$ & 0.37 \\
\hline 6 & 300 & 4.85 & 4.84 & 4.87 & 4.83 & 4.87 & 4.852 & $4.852 \pm 0.32 \%$ & 0.32 \\
\hline 7 & 350 & 5.00 & 4.97 & 4.99 & 5.01 & 5.02 & 4.998 & $4.998 \pm 0.34 \%$ & 0.34 \\
\hline 8 & 400 & 5.10 & 5.17 & 5.19 & 5.06 & 5.10 & 5.124 & $5.124 \pm 0.94 \%$ & 0.94 \\
\hline 9 & 450 & 5.54 & 5.53 & 5.56 & 5.53 & 5.56 & 5.544 & $5.544 \pm 0.24 \%$ & 0.24 \\
\hline 10 & 500 & 5.63 & 5.64 & 5.66 & 5.66 & 5.66 & 5.650 & $5.650 \pm 0.22 \%$ & 0.22 \\
\hline
\end{tabular}

${ }^{1}$ sample number; ${ }^{2}$ glucose concentration; ${ }^{3}$ average of the five experiments; ${ }^{4}$ final average resonance frequency; ${ }^{5}$ relative standard deviation.

Table 3. Performance of the proposed glucose sensor compared with recently reported studies.

\begin{tabular}{cccc}
\hline Ref. & Proposed Method & Response Time (s) & LOD (mg/dL) \\
\hline$[52]$ & $\begin{array}{c}\text { RF patch on silicon substrate by characterization of } \\
\text { effective dielectric constant }\end{array}$ & Not Given & 26.54 \\
\hline$[53]$ & Magnetic acoustic resonance sensor & 22 min & 36.36 \\
\hline$[54]$ & Impedance spectroscopy & Not Given & 12.02 \\
\hline$[55]$ & Forster resonance energy transfer & 15 min & 25 \\
\hline$[56]$ & Rectangular meandered line based RF resonator & 60 & 8.01 \\
\hline$[57]$ & Air bridge enhanced capacitor based RF resonator & 40 & 9.69 \\
\hline This work & hammer-shaped capacitors and spiral inductors \\
constructed RF resonator & $<0.85 \mathrm{~s}$ & 8.01 \\
\hline
\end{tabular}

\section{Conclusions}

Benefiting from an optimized integrated passive device fabrication technique, a novel non-invasive RF glucose sensor was successfully designed, fabricated, and studied with the merits of quantitative detection, robust reusability, ultrafast response time $(<0.85 \mathrm{~s})$ and ultralow LOD $(8.01 \mathrm{mg} / \mathrm{dL})$. Based on the EM simulation result, it is verified that the embedded hammer-shaped capacitors had a fatal effect on the resonance frequency. Meanwhile, the phenomenon that the concentrations of glucose are proportional to biosensor resonance frequency and the magnitude of reflection coefficient enables the proposed chip to accurately detect the glucose levels in diabetes patients. In order to enhance the sensitivity of the biosensor, a $\mathrm{Cl}_{2} /$ Ar plasma-based dry etching treatment with different etching times was carried out, which implies that etching treatment with appropriate time can modify the metal surface to a certain extent. The above findings do not merely reveal several new approaches to realize a stable and accurate platform for the detection of glucose levels but also provide a promising approach for the detection of glucose levels in human serum.

Author Contributions: Conceptualization, Y.L. and Z.Y.; methodology, Y.L. and W.Y.; software, W.Y.; validation, C.Z., S.G. and C.W.; formal analysis, G.S.; investigation, Y.L.; data curation, Z.Y.; writing-original draft preparation, Y.L.; writing-review and editing, Y.Z.; supervision, C.W.; project administration, G.S.; funding acquisition, W.Y. All authors have read and agreed to the published version of the manuscript.

Funding: This work was supported by the National Natural Science Foundation of China under Grant (61805101, 61604060, 61904092), Shandong Provincial Natural Science Foundation of China under Grant (ZR2017JL027, ZR2018BF025, ZR2019BF013, ZR2018BF027), Shandong Province Key Research and Development Program (2019RKB01023), and was also sponsored by Zhejiang Lab (2019MC0AB03).

Conflicts of Interest: The authors declare no competing financial interest. 


\section{References}

1. Fagherazzi, G.; Ravaud, P. Digital diabetes: Perspectives for diabetes prevention, management and research. Diabetes Metab. 2019, 45, 322-329. [CrossRef] [PubMed]

2. Henning, R.J. Type-2 diabetes mellitus and cardiovascular disease. Future Cardiol. 2018, 14, 491-509. [CrossRef]

3. Rawshani, A.; Rawshani, A.; Franzen, S.; Sattar, N.; Eliasson, B.; Svensson, A.M.; Zethelius, B.; Miftaraj, M.; McGuire, D.K.; Rosengren, A.; et al. Risk factors, mortality, and cardiovascular outcomes in patients with Type 2 diabetes. N. Engl. J. Med. 2018, 379, 633-644. [CrossRef]

4. Nealon, R.S.; Howe, P.R.C.; Jansen, L.; Garg, M.; Wong, R.H.X. Impaired cerebrovascular responsiveness and cognitive performance in adults with type 2 diabetes. J. Diabetes Complicat. 2017, 31, 462-467. [CrossRef]

5. Wilkinson, I.D.; Fang, F.; Greig, M.; Hunt, L.; Pallai, S.; Tesfaye, S.; Selvarajah, D. Cerebrovascular status and Type 1 diabetes duration. Diabetic Med. 2018, 35, 86-87.

6. Fradkin, J.E. Confronting the urgent challenge of diabetes: An overview. Health Aff. 2012, 31, 12-19. [CrossRef]

7. Pedraza, E.; Karajic, A.; Raoux, M.; Perrier, R.; Pirog, A.; Lebreton, F.; Arbault, S.; Gaitan, J.; Renaud, S.; Kuhn, A.; et al. Guiding pancreatic beta cells to target electrodes in a whole-cell biosensor for diabetes. Lab Chip 2015, 15, 3880-3890. [CrossRef]

8. Salek-Maghsoudi, A.; Vakhshiteh, F.; Torabi, R.; Hassani, S.; Ganjali, M.R.; Norouzi, P.; Hosseini, M.; Abdollahi, M. Recent advances in biosensor technology in assessment of early diabetes biomarkers. Biosens. Bioelectron. 2018, 99, 122-135. [CrossRef]

9. Geng, Z.X.; Tang, F.; Ding, Y.D.; Li, S.Z.; Wang, X.H. Noninvasive continuous glucose monitoring using a multisensor-based glucometer and time series analysis. Sci. Rep. 2017, 7, 12650. [CrossRef]

10. Li, X.; Li, Y.Q.; Qiu, Q.; Wen, Q.R.; Zhang, Q.; Yang, W.J.; Yuwen, L.H.; Weng, L.X.; Wang, L.H. Efficient biofunctionalization of $\mathrm{MoS}_{2}$ nanosheets with peptides as intracellular fluorescent biosensor for sensitive detection of caspase-3 activity. J. Colloid Interface Sci. 2019, 543, 96-105. [CrossRef]

11. Wang, G.; Qin, J.; Zhao, Y.X.; Wei, J. Nanoporous carbon spheres derived from metal-phenolic coordination polymers for supercapacitor and biosensor. J. Colloid Interface Sci. 2019, 544, 241-248. [CrossRef] [PubMed]

12. Bhat, K.S.; Ahmad, R.; Yoo, J.Y.; Hahn, Y.B. Nozzle-jet printed flexible field-effect transistor biosensor for high performance glucose detection. J. Colloid Interface Sci. 2017, 506, 188-196. [CrossRef] [PubMed]

13. Colombo, L.; Garay, F. A robust and practically free of charge intermittent use glucose biosensor. Sens. Actuators B 2018, 256, 751-759. [CrossRef]

14. Aleeva, Y.; Maira, G.; Scopelliti, M.; Vinciguerra, V.; Scandurra, G.; Cannata, G.; Giusi, G.; Ciofi, C.; Figa, V.; Occhipinti, L.G.; et al. Amperometric biosensor and front-end electronics for remote glucose monitoring by crosslinked PEDOT-glucose oxidase. IEEE Sens. J. 2018, 18, 4869-4878. [CrossRef]

15. Coyle, V.E.; Kandjani, A.E.; Field, M.R.; Hartley, P.; Chen, M.; Sabri, Y.M.; Bhargava, S.K. $\mathrm{Co}_{3} \mathrm{O}_{4}$ needles on $\mathrm{Au}$ honeycomb as a non-invasive electrochemical biosensor for glucose in saliva. Biosens. Bioelectron. 2019, 141, 111479. [CrossRef]

16. Yoon, J.; Lee, S.N.; Shin, M.K.; Kim, H.W.; Choi, H.K.; Lee, T.; Choi, J.W. Flexible electrochemical glucose biosensor based on GOx/gold/MoS $/$ gold nanofilm on the polymer electrode. Biosens. Bioelectron. 2019, 140, 83-89. [CrossRef]

17. Kim, J.; Khan, M.; Park, S. Glucose sensor using liquid-crystal droplets made by microfluidics. ACS Appl. Mater. Interfaces 2013, 5, 13135-13139. [CrossRef]

18. Cardiel, J.J.; Zhao, Y.; Tonggu, L.; Wang, L.; Chung, J.; Shen, A.Q. Flow-induced immobilization of glucose oxidase in nonionic micellar nanogels for glucose sensing. Lab Chip 2014, 14, 3912-3916. [CrossRef]

19. Sorensen, K.T.; Kristensen, A. Label-free monitoring of diffusion in microfluidics. Micromachines 2017, 8, 329. [CrossRef]

20. Jung, D.G.; Jung, D.; Kong, S.H. A lab-on-a-chip-based non-invasive optical sensor for measuring glucose in saliva. Sensors 2017, 17, 2607. [CrossRef]

21. Hu, T.; Ye, Y.; Chen, K.; Long, F.; Sang, W.; Zhou, Y.; Sun, D.; Ni, Z. Visual detection of glucose based on quantum dots aerogel in microfluidic chips. Anal. Methods 2018, 10, 5749-5754. [CrossRef] 
22. Liu, Q.; Liu, Y.; Wu, F.; Cao, X.; Li, Z.; Alharbi, M.; Abbas, A.N.; Amer, M.R.; Zhou, C. Highly sensitive and wearable $\mathrm{In}_{2} \mathrm{O}_{3}$ nanoribbon transistor biosensors with integrated on-chip gate for glucose monitoring in body fluids. ACS Nano 2018, 12, 1170-1178. [CrossRef] [PubMed]

23. Kelemen, L.; Lepera, E.; Horvath, B.; Ormos, P.; Osellame, R.; Vazquez, R.M. Direct writing of optical microresonators in a lab-on-a-chip for label-free biosensing. Lab Chip 2019, 19, 1985-1990. [CrossRef] [PubMed]

24. Bae, C.W.; Toi, T.T.; Kim, B.Y.; Lee, W.I.; Lee, H.B.; Hanif, A.; Lee, E.H.; Lee, N. Fully stretchable capillary microfluidics-integrated nanoporous gold electrochemical sensor for wearable continuous glucose monitoring. ACS Appl. Mater. Interfaces 2019, 11, 14567-14575. [CrossRef]

25. Muniswamy, V.; Pattnaik, P.K.; Krishnaswamy, N. Modeling and analysis of SOI gratings-based opto-fluidic biosensor for lab-on-a-chip applications. Photonics 2019, 6, 71. [CrossRef]

26. Elomaa, J.; Gallegos, L.; Gomez, F.A. Cord-based microfluidic chips as a platform for ELISA and glucose assays. Micromachines 2019, 10, 614. [CrossRef]

27. Muguruma, H.; Hoshino, T.; Nowaki, K. Electronically type-sorted carbon nanotube-based electrochemical biosensors with glucose oxidase and dehydrogenase. ACS Appl. Mater. Interfaces 2015, 7, 584-592. [CrossRef]

28. Parlak, O.; Incel, A.; Uzun, L.; Turner, A.P.F.; Tiwari, A. Structuring Au nanoparticles on two-dimensional $\mathrm{MoS}_{2}$ nanosheets for electrochemical glucose biosensors. Biosens. Bioelectron. 2017, 89, 545-550. [CrossRef]

29. Hughes, G.; Pemberton, R.M.; Nicholas, P.; Hart, J.P. Fabrication of miniaturised screen-printed glucose biosensors, using a water-based ink, and the evaluation of their electrochemical behavior. Electroanalysis 2018, 30, 1608-1612. [CrossRef]

30. Bharath, G.; Madhu, R.; Chen, S.M.; Veeramani, V.; Balamurugan, A.; Mangalaraj, D.; Viswanathan, C.; Ponpandian, N. Enzymatic electrochemical glucose biosensors by mesoporous 1D hydroxyapatite-on-2D reduced graphene oxide. J. Mater. Chem. B 2015, 3, 1360-1370. [CrossRef]

31. Cevik, E.; Cerit, A.; Tombuloglu, H.; Sabit, H.; Yildiz, H.B. Electrochemical glucose biosensors: Whole cell microbial and enzymatic determination based on 10-(4h-dithieno [3,2-b:2 ', 3'-d] pyrrol-4-yl) decan-1-amine interfaced glassy carbon electrodes. Anal. Lett. 2019, 52, 1138-1152. [CrossRef]

32. Jiao, K.L.; Jiang, Y.; Kang, Z.P.; Peng, R.Y.; Jiao, S.Q.; Hu, Z.Q. Three-dimensional $\mathrm{Co}_{3} \mathrm{O}_{4} @ \mathrm{MWNTs}$ nanocomposite with enhanced electrochemical performance for nonenzymatic glucose biosensors and biofuel cells. R. Soc. Open Sci. 2017, 4, 170991. [CrossRef] [PubMed]

33. Gosselin, E.; Eynde, J.J.V.; Petit, A.; Conti, J.; De Coninck, J. Designing a high performance, stable spectroscopic biosensor for the binding of large and small molecules. J. Colloid Interface Sci. 2017, 508, 443-454. [CrossRef]

34. Safaryan, S.M.; Yakovlev, A.V.; Vinogradov, A.V.; Vinogradov, V.V. Inkjet printing of the chromogen free oxidase based optical biosensors. Sens. Actuators B 2017, 251, 746-752. [CrossRef]

35. Crosley, M.S.; Yip, W.T. Kinetically doped silica Sol-Gel optical biosensors: Expanding potential through Dip-Coating. ACS Omega 2018, 3, 7971-7978. [CrossRef]

36. Bai, W.B.; Yang, H.J.; Ma, Y.J.; Chen, H.; Shin, J.; Liu, Y.H.; Yang, Q.S.; Kandela, I.; Liu, Z.H.; Kang, S.K.; et al. Flexible transient optical waveguides and surface-wave biosensors constructed from monocrystalline silicon. Adv. Mater. 2018, 30, e1801584. [CrossRef]

37. Chen, Y.J.; Schoeler, U.; Huang, C.H.; Vollmer, F. Combining whispering-gallery mode optical biosensors with microfluidics for real-time detection of protein secretion from living cells in complex media. Small 2018, 14, 14. [CrossRef]

38. You, K.E.; Uddin, N.; Kim, T.H.; Fan, Q.H.; Yoon, H.J. Highly sensitive detection of biological substances using microfluidic enhanced Fabry-Perot etalon-based optical biosensors. Sens. Actuators B 2018, 277, 62-68. [CrossRef]

39. Guan, B.O.; Huang, Y.Y. Interface sensitized optical microfiber biosensors. J. Lightwave Technol. 2019, 37, $2616-2622$. [CrossRef]

40. Lu, M.D.; Zhu, H.; Bazuin, C.G.; Peng, W.; Masson, J.F. Polymer-templated gold nanoparticles on optical fibers for enhanced-sensitivity localized surface plasmon resonance biosensors. ACS Sens. 2019, 4, 613-622. [CrossRef]

41. Majdinasab, M.; Mitsubayashi, K.; Marty, J.L. Optical and electrochemical sensors and biosensors for the detection of quinolones. Trends Biotechnol. 2019, 37, 898-915. [CrossRef] 
42. Deshours, F.; Alquie, G.; Kokabi, H.; Rachedi, K.; Tlili, M.; Hardinata, S.; Koskas, F. Improved microwave biosensor for non-invasive dielectric characterization of biological tissues. Microelectron. J. 2019, 88, 137-144. [CrossRef]

43. Zhang, R.; Chen, Q.M.; Liu, K.; Chen, Z.F.; Li, K.D.; Zhang, X.M.; Xu, J.B.; Pickwell-MacPherson, E. Terahertz microfluidic metamaterial biosensor for sensitive detection of small-volume liquid samples. IEEE Trans. Terahertz Sci. Technol. 2019, 9, 209-214. [CrossRef]

44. Liu, G.; Cheng, D.; Zhang, B.; Shu, G.X.; Wang, J.X. A microwave biosensor based on spoof surface plasmon polaritons for in vivo measurement of the water content of human skin tissues. J. Phys. D Appl. Phys. 2019, 52, 205401. [CrossRef]

45. Narang, R.; Mohammadi, S.; Ashani, M.M.; Sadabadi, H.; Hejazi, H.; Zarifi, M.H.; Sanati-Nezhad, A. Sensitive, real-time and non-intrusive detection of concentration and growth of pathogenic bacteria using microfluidic-microwave ring resonator biosensor. Sci. Rep. 2018, 8, 15807. [CrossRef]

46. Liu, C.F.; Wang, M.H.; Jang, L.S. Microfluidics-based hairpin resonator biosensor for biological cell detection. Sens. Actuators B 2018, 263, 129-136. [CrossRef]

47. Matsunaga, M.; Kobayashi, A.; Nakazato, K.; Niitsu, K. Design trade-off between spatial resolution and power consumption in CMOS biosensor circuit based on millimeter-wave LC oscillator array. J. Phys. D Appl. Phys. 2018, 57. [CrossRef]

48. Hong, J.S.; Lancaster, M.J. Microstrip Filters for RF/Microwave Applications; John Wiley \& Sond. Inc.: Hoboken, NJ, USA, 2001.

49. Wang, C.; Lee, W.S.; Zhang, F.; Kim, N.Y. A novel method for the fabrication of integrated passive device on SI-GaAs substrate. Int. J. Adv. Manuf. Tech. 2011, 52, 1011-1018. [CrossRef]

50. Li, Y.; Wang, C.; Kim, N.Y. A high performance compact Wilkinson power divider using GaAs-based optimized integrated passive device fabrication process for LTE application. Solid State Electron. 2015, 103, 147-153. [CrossRef]

51. Yoon, G. Dielectric properties of glucose in bulk aqueous solutions: Influence of electrode polarization and modeling. Biosens. Bioelectron. 2011, 26, 2347-2353. [CrossRef]

52. Qiang, T.; Wang, C.; Kim, N.Y. Quantitative detection of glucose level based on radiofrequency patch biosensor combined with volume-fixed structures. Biosens. Bioelectron. 2017, 98, 357-363. [CrossRef] [PubMed]

53. Hu, R.; Stevenson, A.C.; Lowe, C.R. An acoustic glucose sensor. Biosens. Bioelectron. 2012, 35, 425-428. [CrossRef] [PubMed]

54. Kim, D.; Kim, M.; Reddy, S.S.; Cho, J.; Cho, C.; Jung, S.; Shim, Y. Electron-transfer mediator for a NAD-glucose dehydrogenase-based glucose sensor. Anal. Chem. 2013, 85, 11643-11649. [CrossRef] [PubMed]

55. Aloraefy, M.; Pfefer, T.J.; Ramella-Roman, J.C.; Sapsford, K.E. In vitro evaluation of fluorescence glucose biosensor response. Sensors 2014, 14, 12127-12148. [CrossRef]

56. Kim, N.Y.; Dhakal, R.; Adhikari, K.K.; Kim, E.S.; Wang, C. A reusable robust radio frequency biosensor using microwave resonator by integrated passive device technology for quantitative detection of glucose level. Biosens. Bioelectron. 2015, 67, 687-693. [CrossRef]

57. Dhakal, R.; Wang, C.; Kim, E.S.; Kim, N.Y. Complex permittivity characterization of serum with an air-bridge enhanced capacitor for quantifiable detection of glucose. Appl. Phys. Lett. 2015, 106, 073702. [CrossRef]

(C) 2020 by the authors. Licensee MDPI, Basel, Switzerland. This article is an open access article distributed under the terms and conditions of the Creative Commons Attribution (CC BY) license (http://creativecommons.org/licenses/by/4.0/). 